\title{
ARTICLE CmFTL2 is involved in the photoperiod- and sucrose-mediated
control of flowering time in chrysanthemum
}

Jing Sun ${ }^{1,2}$, Heng Wang ${ }^{1,2}$, Liping Ren ${ }^{1}$, Sumei Chen ${ }^{1}$, Fadi Chen ${ }^{1}$ and Jiafu Jiang ${ }^{1}$

The chrysanthemum genome harbors three FT-like genes: CMFTL1 and CMFTL3 are thought to act as regulators of floral induction under long-day (LD) and short-day (SD) conditions, respectively, whereas the function of CmFTL2 is currently unclear. The objective of the present research was to explore the function of CMFTL2 in the determination of flowering time of the photo-insensitive chrysanthemum cultivar 'Floral Yuuka', both in response to variation in the photoperiod and to the exogenous provision of sucrose. Spraying leaves of 'Floral Yuuka' plants with $50 \mathrm{mM}$ sucrose accelerated flowering and increased the level of CmFTL2 transcription in the leaf more strongly than either CMFTL1 or FTL3 under both long and SD conditions. Transcription profiling indicated that all three CmFTL genes were upregulated during floral induction. The relationship of the CmFTL2 sequence with that of other members of the PEBP family suggested that its product contributes to the florigen rather than to the anti-florigen complex. The heterologous expression of CmFTL2 in the Arabidopsis thaliana ft-10 mutant rescued the mutant phenotype, showing that CmFTL2 could compensate for the absence of FT. These results suggest that CMFTL2 acts as a regulator of floral transition and responds to both the photoperiod and sucrose.

Horticulture Research (2017) 4, 17001; doi:10.1038/hortres.2017.1; Published online 15 February 2017

\section{INTRODUCTION}

The switch from vegetative to reproductive growth is a key event in the life cycle of plants. This switch is triggered by a variety of both environmental cues (notably photoperiod and temperature) and endogenous signals (hormonal status and carbohydrate availability) sensed by the shoot apical meristem. ${ }^{1-4}$ A change in the photoperiod stimulates a network of regulatory genes, one of the most important of which is FT. FT integrates signals from various relevant pathways ${ }^{5}$ and is transported via the phloem to the shoot apex. ${ }^{6-9}$ The protein encoded by $F T$ belongs to a small family whose members share homology with the mammalian phosphatidyl ethanolamine-binding proteins (PEBPs). The genome of the model angiosperm Arabidopsis thaliana harbors, in addition to $F T$, the related genes TSF, BFT, ATC, MFT and $T F L 1$, all of which are involved in floral induction. ${ }^{5,10-13} F T$ or FT-like genes have been described in a growing number of plant species, including the chrysanthemum. ${ }^{14-22}$

Sugars not only represent the primary source of carbon and energy but also act as regulator molecules controlling metabolism, the stress response, growth and development. Sucrose, the predominant form of sugar in plant tissue, has been shown to promote flowering in various plant species. ${ }^{3,21,22}$ Shortly before floral initiation in $A$. thaliana plants exposed to SD conditions, the level of both sucrose and gibberellins $\left(\mathrm{GA}_{4}\right)$ in the shoot apex increases markedly. ${ }^{23}$ In Sinapis alba, phloem sap sucrose levels rise during floral induction under both displaced SD and LD conditions. ${ }^{24}$ In Xanthium strumarium, exposing a leaf to a single inductive photoperiod rapidly increases its sucrose content. ${ }^{25}$ Nevertheless, there is no evidence for the accumulation of sucrose during floral transition, suggesting that its role in the flowering process is to contribute to sucrose homeostasis in a speciesspecific manner. ${ }^{3}$ A genetic analysis has shown that in A. thaliana, loci associated with the determination of flowering time map to the same genomic region as those determining the content of certain carbohydrates, a coincidence that has been taken to imply a functional relationship between carbohydrate levels and flowering time. ${ }^{26}$ Sugars present in the leaf can act as a mobile signal to raise the level of SQUAMOSA PROMOTER BINDING PROTEIN-LIKE, thereby triggering floral transition in A. thaliana. ${ }^{27}$

Chrysanthemum is a popular ornamental species, second only to the rose. ${ }^{28}$ The summer-to-autumn flowering cultivar 'Floral Yuuka' flowers under both LDs and SDs, although it flowers earlier under SD conditions. RNA-seq analysis has suggested the involvement of the sugar signaling pathway in its floral transition under LD conditions. ${ }^{29}$ CSFTL3 is a key regulator of photoperiodinduced flowering under short days in chrysanthemum, ${ }^{30}$ whereas CSFTL1 is probably required for flowering under LDs. ${ }^{31}$ The function of CSFTL2 has not yet been defined. Here, the CMFTL copies present in 'Floral Yuuka' were characterized. In particular, transcriptional profiling was used to show that the CMFTL2 transcript accumulates during floral transition in plants exposed to both SDs and LDs. The abundance of the transcript is increased when the plants are provided with exogenous sucrose, a treatment that also accelerates flowering. The heterologous expression of CmFTL2 in A. thaliana rescues the phenotype of an $F T$ loss-of-function mutant. Our results suggest that the involvement of CMFTL2 in floral induction operates through one or more photoperiod- and sucrose-regulated pathways.

\section{MATERIALS AND METHODS}

Plant materials and growing conditions

Chrysanthemum (cultivar 'Floral Yuuka') plants were maintained by the Chrysanthemum Germplasm Resource Preserving Centre (Nanjing 
Agricultural University, China). Cuttings of uniform height $(6-7 \mathrm{~cm})$ and vigor were selected for the present experiments and were rooted and grown in a greenhouse under a $16 \mathrm{~h}$ photoperiod provided by $300 \mu \mathrm{E} \mathrm{m} \mathrm{m}^{-2}$ $\mathrm{s}^{-1}$ lighting. The lit period temperature was kept in the range of $21-25^{\circ} \mathrm{C}$, and the dark period temperature was maintained at $15-17^{\circ} \mathrm{C}$.

The isolation of CMFTL1, CmFTL2 and CMFTL3 CDNAs and a phylogenetic analysis of their sequences

Total RNA was extracted from snap-frozen leaves using a Plant RNA Extraction Kit (Takara Bio, Dalian, China), following the manufacturer's protocol. A $1 \mu \mathrm{g}$ aliquot was reverse-transcribed in a $10 \mu \mathrm{L}$ reaction volume based on the Superscript First-Strand Synthesis System (Invitrogen, Carlsbad, CA, USA). For the subsequent amplification of the CmFTL open reading frame (ORF) sequences, a $1 \mu \mathrm{L}$ aliquot of the CDNA preparation (equivalent to $100 \mathrm{ng}$ ) was used as the template in reactions primed by oligomers targeting CMFTL1, CMFTL2 or CmFTL3. ${ }^{30}$ The PCR protocol comprised a $94^{\circ} \mathrm{C} / 3$ min denaturation, followed by 32 cycles of $94^{\circ} \mathrm{C} / 30 \mathrm{~s}$, $55^{\circ} \mathrm{C} / 30 \mathrm{~s}$ and $72{ }^{\circ} \mathrm{C} / 60 \mathrm{~s}$ and was completed by a $72{ }^{\circ} \mathrm{C} / 7$ min extension. The amplicons were introduced separately into the pMD19-T Simple Vector (Takara Bio) for sequencing. The remainder of each CDNA sequence was acquired using RACE-PCR following methods described elsewhere. ${ }^{32}$ The deduced polypeptide sequences were subjected to a BLASTp search (www. ncbi.nlm.nih.gov/blast/) to identify homologs, and the members of the PEBP family were clustered using Clustal X software (www.clustal.org/ clustal2/). A neighbor-joining tree was generated using MEGA 5 software (www.megasoftware.net), applying the Poisson model with gammadistributed rates and 1000 bootstrap replicates.

Semi-quantitative RT-PCR and real-time quantitative RT-PCR

A $1 \mu \mathrm{g}$ aliquot of total leaf RNA, extracted as described above, was reversetranscribed in a $10 \mu \mathrm{L}$ reaction volume using the Superscript First-Strand Synthesis System (Invitrogen). Real-time quantitative RT-PCR (qRT-PCR) analyses were conducted in accordance with MIQE guidelines. ${ }^{33}$ The primers targeting the three CMFTL genes (sequences presented in Supplementary Table S1) were designed based on the sequence close to the $3^{\prime}$ end of each gene, including the $3^{\prime}-U_{T R} .^{30}$ All amplicons were sequenced to confirm their identities. The qRT-PCRs were performed using a SYBR Premix Ex Taq Kit (Takara Bio), in accordance with the manufacturer's protocol. The PCR protocol comprised a $95^{\circ} \mathrm{C} / 2 \mathrm{~min}$ denaturation, followed by 40 cycles of $95^{\circ}$ $\mathrm{C} / 15 \mathrm{~s}, 55^{\circ} \mathrm{C} / 15 \mathrm{~s}$, and $68^{\circ} \mathrm{C} / 20 \mathrm{~s}$. The products were subjected to a melting curve analysis. The EF1a gene ${ }^{30}$ was used as the reference sequence.

\section{Temporal profiling of CmFTL transcription}

A set of 300 rooted 'Floral Yuuka' cuttings of uniform length was grown in a greenhouse maintained at a temperature of $\sim 22^{\circ} \mathrm{C}$ under an $8 \mathrm{~h}$ photoperiod (hereafter, SD), including a $4 \mathrm{~h}$ night break (NB) (between 22:00 and 02:00) provided by high-pressure sodium lumps. The SD+NB regime was maintained until the floral bud was visible in the plants. The third leaf below the apex of three plants was harvested at 16:00 every other day between days 67 and 104 (flowering stage) based on a previously described method. ${ }^{34}$ A second set of 300 cuttings was exposed to SD+NB up to day 73 , then to SD; leaves were sampled from these plants every other day at 16:00 between days 73 and 88. RNA was extracted from snap-frozen leaf samples, as described above. The experiment was replicated at least twice. Shoot apices sampled at various developmental stages were fixed in formalin: acetic acid: alcohol (v/v, 5:5:90), embedded in Paraplast (Sigma, Shanghai, China), cut into $10 \mu \mathrm{m}$ sections and stained with $1 \% \mathrm{w} / \mathrm{v}$ toluidine blue.

\section{The induction of flowering by sucrose treatment}

A set of 300 'Floral Yuuka' cuttings was grown under an SD+NB regime up to the 15 leaf stage, at which point three equal groups of 100 plants each were formed. One group received an application of aqueous $50 \mathrm{mM}$ sucrose sprayed onto their leaves every four days; a second group was simultaneously sprayed with water; plants' leaves in the third group was semi-defoliated. We sectioned the stem tip by hand and examined it using a stereomicroscope. We used the time when the shoot apical meristems begin to expand as the indicator of floral bud initiation.

\section{The transcriptional response to sucrose treatment}

One hundred of 'Floral Yuuka' rooted cuttings of height $10 \mathrm{~cm}$ were grown under a SD $+N B$ (light period $23^{\circ} \mathrm{C}$, dark period $20^{\circ} \mathrm{C}$ ) regime for 20 days, by which time they had developed $\sim 10$ leaves. Fifty plants were switched to a SD $\left(8 \mathrm{~h}\right.$ photoperiod, lit period $23^{\circ} \mathrm{C}$, dark period $\left.20^{\circ} \mathrm{C}\right)$ regime. After three additional days, the transcriptional response of the CmFTLs to sucrose treatment was examined by first withholding water for seven days and then spraying to dripping with aqueous $50 \mathrm{mM}$ sucrose; a control set of plants was sprayed with water. The third leaf below the apex was harvested at various time points and analyzed by qRT-PCRs targeting the CmFTLs, following the procedures described above for RNA extraction and reverse transcription.

Functional complementation of the $A$. thaliana $\mathrm{ft}-10$ mutation by CMFTL2

The $6977 \mathrm{bp}$ A. thaliana FT promoter from the pENTR4-AtFT promoter vector was introduced into the GATEWAY vector pHGW using LR Clonase II enzyme mix (Invitrogen) to produce the construct pHGW-proFT. ${ }^{35}$ The fulllength CMFTL2 coding sequence was introduced into the recombined pHGW-proFT plasmid at its Xhol and Pstl cloning sites to produce the construct proFT::CMFTL2. The A. thaliana $\mathrm{ft}-10$ mutant ${ }^{36}$ was transformed using the floral dip method. ${ }^{37}$ Two independent transgenic lines were selected by regenerating plants on a Murashige and Skoog (1962) medium containing $25 \mathrm{mg} \mathrm{L}^{-1}$ hygromycin. The presence of the transgene was confirmed by an RT-PCR analysis of RNA extracted from leaves, using primers directed to the CmFTL2 ORF (Supplementary Table S1). A. thaliana ACT2 (At3g18780) was used as the reference sequence. The phenotypic effect of the transgene was recorded in the $T_{1}$ generation. Significance testing was performed using SPSS 20.0 (http://www-01.ibm.com/software/ cn/analytics/spss/).

\section{RESULTS}

\section{Sucrose promoted early flowering in 'Floral Yuuka'}

The effect of sucrose on floral transition in 'Floral Yuuka' was examined by growing plants under a SD plus night break (SD+NB) regime and then spraying them with a sucrose solution. Floral buds became visible on the sucrose-treated plants 34 days after the start of the treatment, and every plant had reached this stage by 38 days (Figure $1 \mathrm{~b}$ ). By contrast, at that time, only $70 \%$ of the water-treated plants and $40 \%$ of the semi-defoliated plants showed visible buds. After an additional 14 days, the proportion of plants in either of these control treatments that had reached flowering was still not $100 \%$. Under SD conditions, there was no discernible treatment effect, as all three sets of plants reached flowering at the same time (Figure 1b). Thus, the sucrose treatment clearly accelerated flowering in 'Floral Yuuka' plants

a

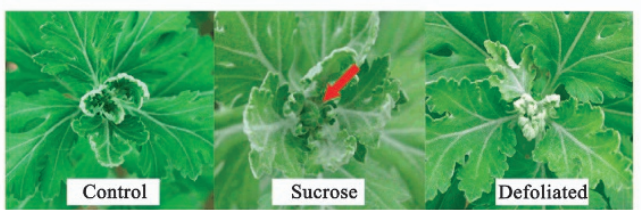

b

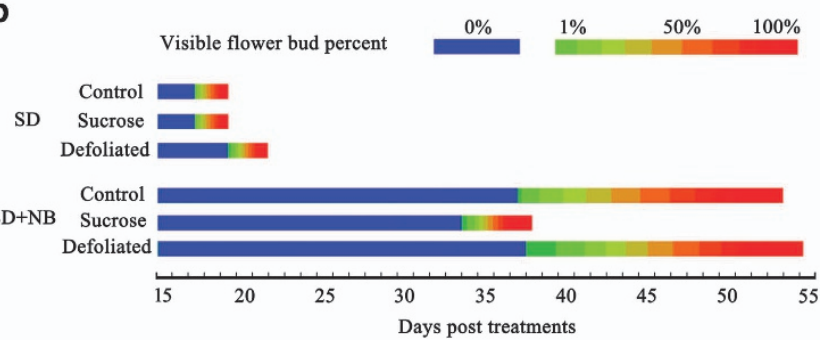

Figure 1. The effect of spraying the leaves of 'Floral Yuuka' plants with a sucrose solution. (a) Floral initiation in plants grown under an $\mathrm{SD}+\mathrm{NB}$ regime. Flower buds are arrowed. (b) The proportion of plants forming flower buds in response to various treatments $(n=100)$. 
a

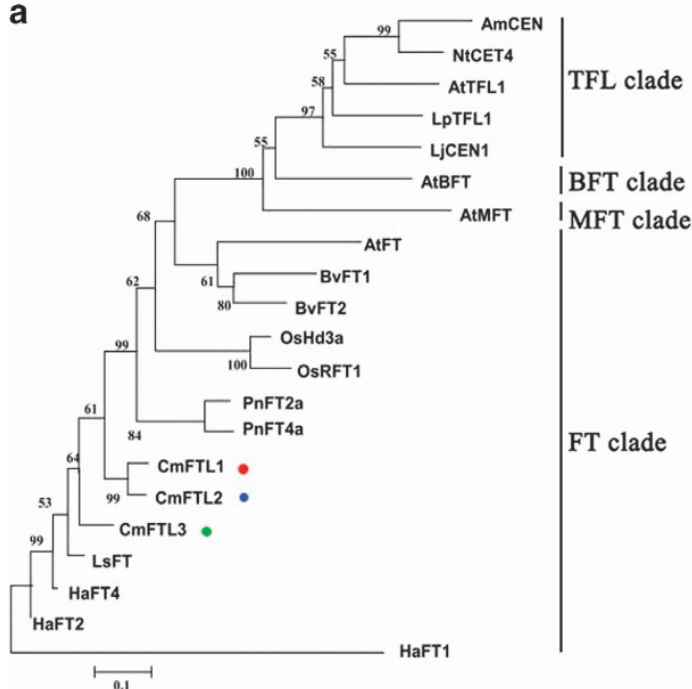

b

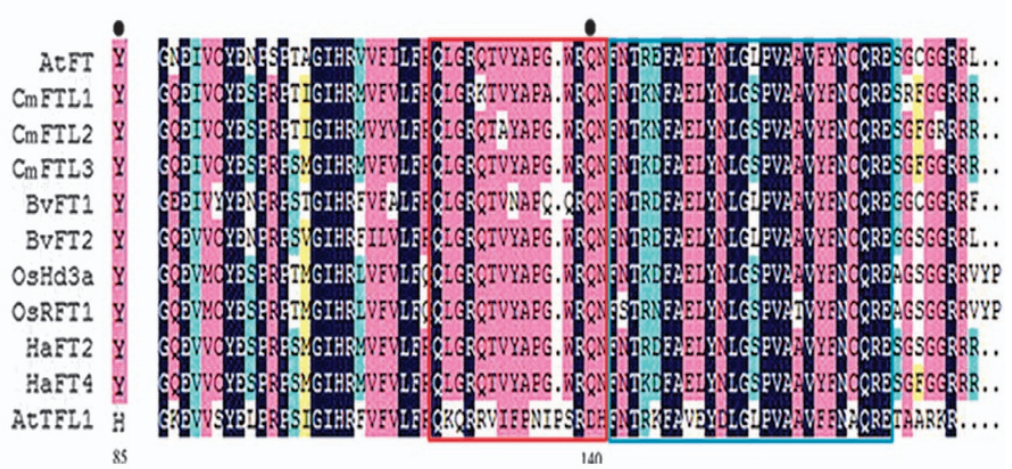

Figure 2. CmFTL polypeptide sequences. (a) Phylogeny of the PEBP family: A. thaliana AtFT (AT1G65480), AtBFT (AT5G62040), AtMFT (AT1G18100), AtTFL1 (NP_196004), Antirrhinum majus AmCEN (Q41261.1), Populus nigra PnFT2a (AB109804.1), PnFT4a (AB369074.1), Lactuca sativa LsFT (BAK14368.1), Chrysanthemum seticuspe CsFTL1 (AB679270), CsFTL2 (AB679271), CsFTL3 (AB679272), rice OsHd3a (JX472280), OsRFT1 (AB426873.1), tobacco NtCET4 (AF145261), Lotus japonicus LpTFL1 (AF316419), LjCEN1 (AY423715), Helianthus annuus HaFT2 (GQ884987.1), HaFT4 (GU985573.1), Beta vulgaris BvFT1 (HM448910.1), and BVFT2 (HM448912.1). (b) Partial alignment of PEBP polypeptides. Filled circles indicate residues present in FT polypeptides but not in FT-like. Y85 and Q140 are essential for the formation of an external loop. The red and blue boxes highlight the conserved segments ( $B$ and $C$ ), required for FT-like activity.

grown under an SD+NB regime. In addition, we also performed the same sucrose treatment on 'Jimba', a short-day cultivar. We found no differences between the experimental group (sucrosetreated) and the control group (water-treated) in terms of the floral bud differentiation time, under both LD and SD conditions. Neither group bolted under the LD conditions (data not shown).

The 'Floral Yuuka' CmFTL alleles

A BLASTp search of the NCBI database showed that the 'Floral Yuuka' CmFTL1, CmFTL2 and CMFTL3 alleles each encoded the same polypeptide as their equivalent in Chrysanthemum seticuspe. ${ }^{30} \mathrm{~A}$ cross-species phylogenetic analysis of PEBP family members indicated that all three genes belong to the clade that includes $F T$ (Figure 2a). Each sequence encoded the residues Y85 and Q140, which are required for the formation of an external loop, and the conserved B and C segments (Figure $2 \mathrm{~b}$ ).

Temporal profiling of CmFTL2 transcription

When 'Floral Yuuka' plants were grown under the SD+NB regime for 116 days, the abundance of each of the three CMFTL transcripts increased between days 67 and 75 (Figure 3a). Following the appearance of the floral primordia, CmM111 (which shares the same ORF as the AP1 homolog CsM111 (AY173054) was upregulated. ${ }^{38}$ The shoot apical leaf buds had reached the doming stage by day 79 , and the shoot apical meristem's involucres had become fully formed by day 88 . Plants that were no longer exposed to NB after day 73 (the SD treatment) reached the doming stage within an additional four days (day 77). Under these conditions, the flowering process was accelerated, with the first florets making their appearance on day 88 (Figure 3b). During the period of exposure to short days, CMFTL2 was upregulated, whereas CmFTL3 was upregulated to a greater extent than CmFTL1. Thus, CmFTL2 transcription occurred during the period of floral transition under both SD+NB and SD conditions.

Expression patterns of CmFTLs

To investigate the effect of light on CMFTL expression in 'Floral Yuuka', the expression patterns of the CmFTLs in leaves of
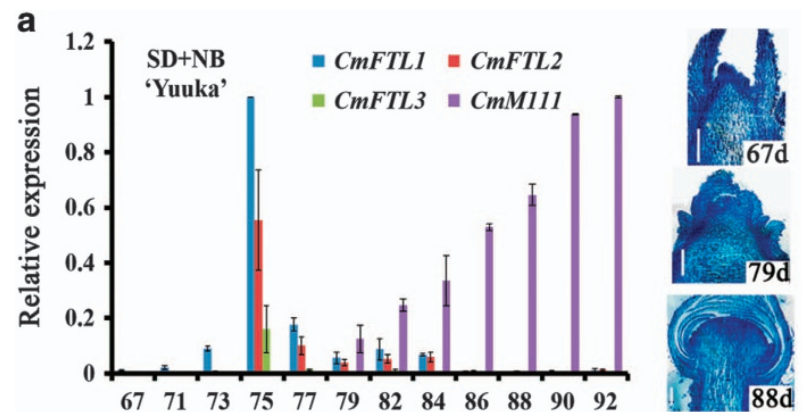

b

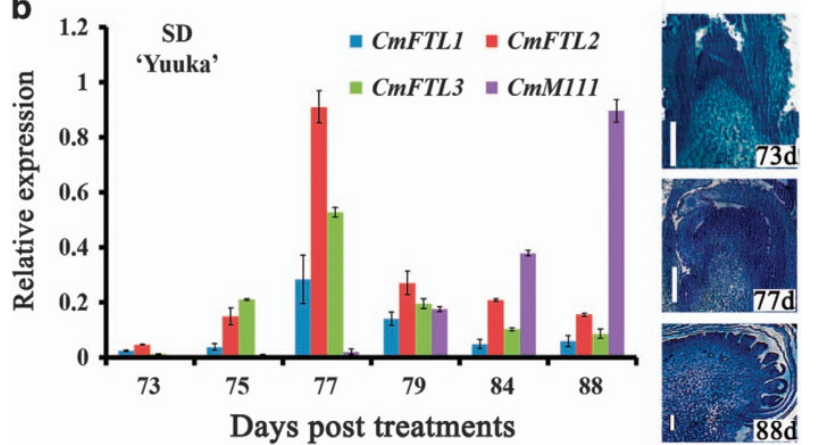

Figure 3. Temporal transcription of CmFTLs in 'Floral Yuuka', as assayed by qRT-PCR. Plants were grown under (a) an SD+NB or (b) an SD regime. Values reflect the mean of three biological replicates, with three technical replicates per biological replicate. The right panel illustrates shoot apices harvested at various developmental stages, embedded in Paraplast and stained with toluidine blue; bar: $1 \mathrm{~mm}$.

'Floral Yuuka' under different light conditions were examined (Figure 4a). All three CmFTL transcript levels were higher during the dark period compared with the light period. Compared with SD conditions, the transcript levels of CMFTL1 were much 
a

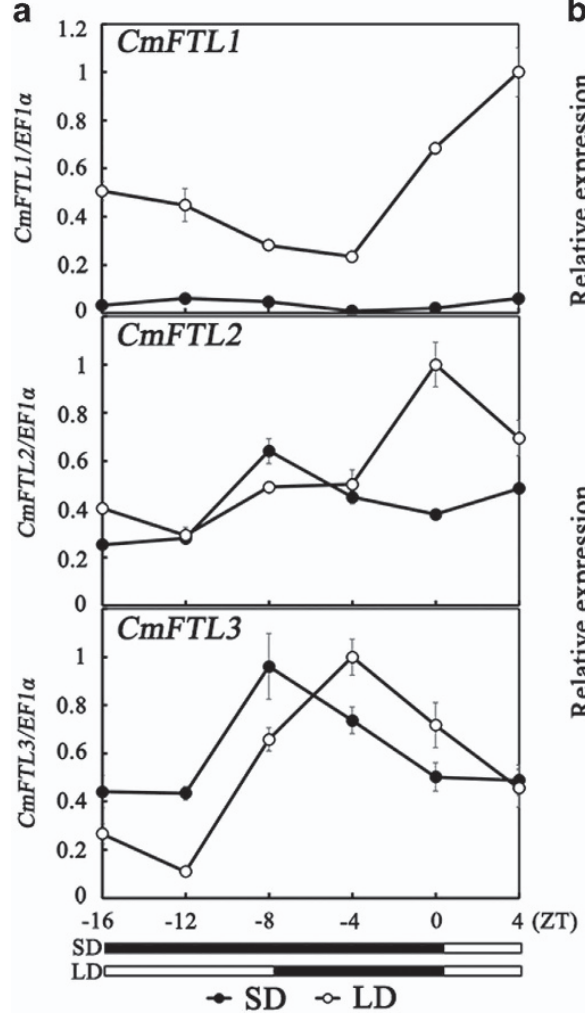

b
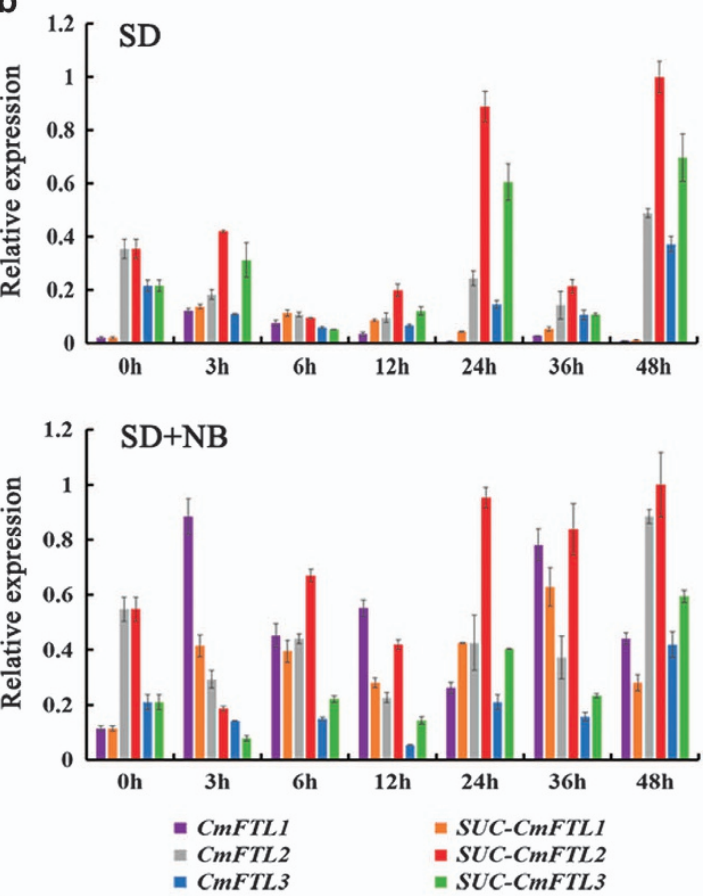

Figure 4. Diurnal variation in CmFTL transcription in 'Floral Yuuka' plants, as assayed by qRT-PCR. (a) Plants exposed to either an LD or an SD regime. The illumination regime is illustrated by white (lit) and black (dark) bars. (b) Plants sprayed with 50 mM sucrose and exposed to either an SD+NB or an SD regime. Values reflect the mean of three biological replicates.

higher under LD conditions. The CMFTL2 transcript levels increased at the start of the dark period and peaked around the middle of the dark period under the SD conditions and at dawn under the LD conditions. The CMFTL3 transcript levels peaked around the middle of the dark period under both SD and LD conditions.

Sucrose treatment induced CMFTL2 transcription

In plants exposed to either SD or SD+NB, CmFTL2 transcription levels were strongly induced by sucrose (Figure 4b). CmFTL2 and CmFTL3 were both transcribed during the SD+NB treatment, with the former accumulating to a higher level than the latter, whereas the level of CMFTL1 transcription declined during this period (Figure 4b). Thus, supplying sucrose exogenously had the effect of inducing CmFTL2 and CMFTL3 in the SD+NB treatment, but CMFTL1 transcription was much more strongly influenced in the $\mathrm{SD}$ than in the SD+NB treatment.

CMFTL2 rescues the phenotype of the $A$. thaliana $\mathrm{ft}-10$ mutant Flowering is delayed in the $A$. thaliana $\mathrm{ft}-10$ mutant. $^{39,40}$ The native AtFT promoter was used to drive CMFTL2 expression in a transformed $\mathrm{ft}-10$ mutant background to confirm its florigenic activity. In both independent transgenic lines, the flowering phenotype reverted to wild type, showing that CMFTL2 was able to fully compensate for the absence of $F T$ (Figure $5 \mathrm{a}$ ). The number of rosette leaves formed per plant was $8-11$ in the transgenic plants but 30-35 in the non-transgenic $\mathrm{ft}-10$ mutant (Figure $5 \mathrm{~b}$ ). A semi-quantitative RT-PCR assay applied to leaves harvested at the flowering stage confirmed the successful transcription of CMFTL2 under LD conditions (Figure 5c).

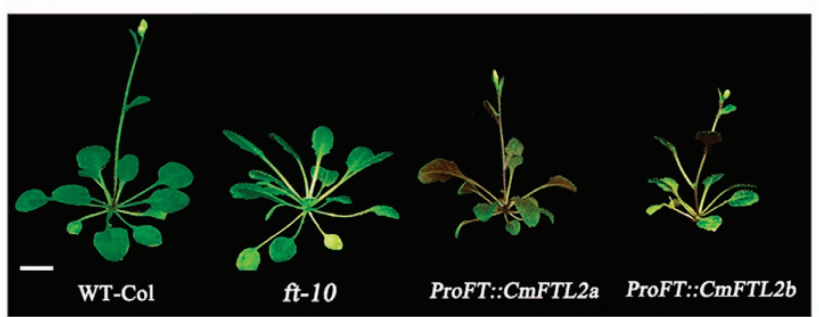

b

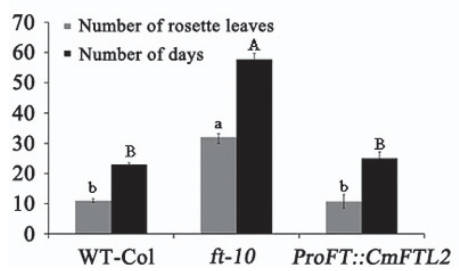

C

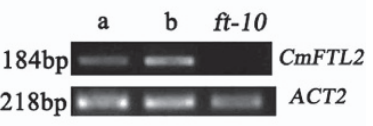

Figure 5. CmFTL2 rescues the phenotype of the $A$. thaliana $\mathrm{ft}-10$ mutant. (a) Plants grown under an SD+NB regime. WT, wild type $A$. thaliana; proFT::CmFTL2a and $b$ : two independent transgenic lines heterologously expressing CmFTL2 in an ft-10 mutant background; bar: $1 \mathrm{~cm}$. (b) The number of rosette leaves and number of days required to reach flowering. Different letters show significant differences. Lowercase and uppercase letters represent the number of days and rosette leaves, respectively. Significance testing for both was performed at the 0.01 level. (c) Semi-quantitative RT-PCR analysis of CMFTL2 transcription at the flowering stage. a and b: two independent transgenic lines heterologously expressing CmFTL2 in an $\mathrm{ft}-10$ mutant background. 


\section{DISCUSSION}

At present, the uniformity of flowering time in summer-to-autumn type chrysanthemum is very important but difficult to control. Three CSFTL genes have been reported in chrysanthemum (a typical SD plant), and CSFTL3 is a key regulator of photoperiodinduced flowering under short days in chrysanthemum. ${ }^{30}$ On the basis of over-expression experiments, the product of CSFTL1 shows weak, florigenic activity. The gene is also induced by LD conditions. Hence, it has been proposed that CSFTL1 encodes a LD florigen equivalent to RFT1. ${ }^{31}$ Here, the transcription of CMFTL1 was higher than that of CmFTL3 under the SD+NB condition in 'Floral Yuuka' (Figure 4b), which is consistent with CSFTL1 in 'Jimba'. ${ }^{31}$ We also wondered whether CMFTL2 in SD and summerto-autumn type chrysanthemums shows different photoperiod responses. The present comparison between the transcriptional behavior of 'Floral Yuuka' (summer-to-autumn type) raised under either an SD or an SD+NB regime showed that the abundance of the CMFTL2 transcript increased in conjunction with floral initiation (Figure 3), suggesting that CmFTL2 had a role during floral transition, which was inconsistent with the studies of SD chrysanthemum. ${ }^{30}$ Once the plants had experienced the SD+NB regime, the abundance of the CMFTL1 transcript increased above that of CMFTL3. In addition, the gene was suppressed by the sucrose treatment. By contrast, the CMFTL2 transcript continued to accumulate in sucrose-treated plants growing under either an SD or an SD+NB regime (Figure 4b), suggesting that in 'Floral Yuuka', both photoperiod and sucrose influence the transcription of the CmFTLs. Meanwhile, the expression of CMFTL2 before and after the sucrose treatment in 'Floral Yuuka' could provide an improved understanding of the exquisite coordination that exists within the photoperiodic and sucrose-induced flowering gene network not only for chrysanthemum but also for various other plant species.

Sugar signaling is important for a wide range of developmental processes, including the regulation of floral induction. ${ }^{27,41,42}$ The most common sugar synthesized by plants is sucrose, which is a more stable molecule than either of its monosaccharide components glucose and fructose, making it a readily transportable compound. In photo-sensitive plants, the exposure of a leaf to a single inductive photoperiod results in a rapid increase in leaf sucrose content. ${ }^{25}$ Sucrose supply to the aerial portion of darkgrown plants does promote flowering in $A$. thaliana. ${ }^{43}$ In LD-grown $A$. thaliana plants, sucrose generated from photosynthetic activity induces the accumulation of the SPL transcript via the down-regulation of miR156 27,42 and amplifies the expression of $F T^{44}$ Because the transition from vegetative to reproductive development is tightly controlled, the flowering response of chrysanthemum cannot be fully explained by a photoperiodbased transcriptional regulation of florigen. ${ }^{31}$ We also performed a transcriptomic analysis. Our results suggested that while the photoperiod and the gibberellin pathways act in concert to promote the flowering of 'Floral Yuuka' plants grown under short days, sugar signaling is more important under LD conditions (data not shown). ${ }^{29}$ These data suggest that sucrose signaling may not be an important floral induction mechanism in 'Floral Yuuka' plants under short days.

In the last common ancestor of mono- and dicotyledonous species, PEBP family proteins include FT-like (encoding floral activators), TFL1-like (encoding floral inhibitors) and MFT-like (encoding factors involved in germination). ${ }^{45}$ The diversification of TFL from FT is thought to have occurred at least 150 mya (Figure 2a). ${ }^{46}$ The current evidence seems to indicate that the emergence of the FT-like genes coincided with the evolution of the flowering plants. ${ }^{47}$ In $A$. thaliana, the variable segment $B$ and one ligand-binding pocket are both essential for FT function, whereas FT-like function requires segments B and C (Figure $2 \mathrm{~b}$ ). ${ }^{48}$ The segment B residues Q140 and D144 contribute to the function of both FT and TF-like1. Alignment of FT fourth exon sequences demonstrated that Q140 was present in CMFTL2, which suggests that CmFTL2, like AtFT, forms the external loop that provides an anion-binding site (Figure $2 \mathrm{~b}$ ). The implication is that the Y 85 and Q140 residues allow CmFTL2 to act as a florigen mediating the onset of flowering in chrysanthemum. The likelihood is that CMFTL2 contributes to the florigen rather than the anti-florigen complex. The three CmFTL products all appear to be involved in the regulation of flowering time. However, both the nature of the cross-talk between photoperiod and endogenous sucrose level during floral induction and how the CmFTLs function in the regulatory network currently remain unknown. The results from this study suggest that the existence of a sugar-induced CMFTL pathway cannot be excluded and might play an important role in the photoperiod- and sucrose-mediated control of flowering time in chrysanthemum. Further investigations to determine the role of CmFTL genes in the growth and flowering of chrysanthemums will provide clues for a better understanding of this process.

\section{Conclusions}

The rescue of the delayed flowering phenotype of the $A$. thaliana $\mathrm{ft}-10$ mutant by CMFTL2 confirmed that CMFTL2 is a florigen. CMFTL2 was active during the process of floral transition under an $\mathrm{SD}$ or an SD+NB regime and much more strongly induced than either CMFTL1 or CMFTL3 by sucrose, which might be involved in the flowering of 'Floral Yuuka'.

\section{Availability of data and materials}

All the data supporting our findings are contained within the manuscript, in text, tables and figures and in the Supplementary Files.

\section{CONFLICT OF INTEREST}

The authors declare no conflict of interest.

\section{ACKNOWLEDGEMENTS}

We thank Dr Yuehui He (National University of Singapore) for providing the pENTR4AtFT promoter vector and seeds of the Arabidopsis ft-10 mutant. This work was supported by funding from the National Natural Science Foundation of China (31372100), the Project Funded by the Priority Academic Program Development of Jiangsu Higher Education Institutions, and by 'Programs of Innovation and Entrepreneurship Talents' of Jiangsu Province.

\section{AUTHOR CONTRIBUTIONS}

JS, HW and LR performed the experiments; JS wrote the manuscript; SC and FC revised the manuscript; $J$ J designed the experiments.

\section{REFERENCES}

1 Fornara F, de Montaigu A, Coupland G. SnapShot: control of flowering in Arabidopsis. Cell 2010; 141: e551-e552.

2 Simpson GG, Dean C. Arabidopsis, the Rosetta stone of flowering time? Science 2002; 296: 285-289.

3 Srikanth A, Schmid M. Regulation of flowering time: all roads lead to Rome. Cell Mol Life Sci 2011; 68: 2013-2037.

4 Thomas B. Light signals and flowering. J Exp Bot 2006; 57: 3387-3393.

5 Turck F, Fornara F, Coupland G. Regulation and identity of florigen: FLOWERING LOCUS T moves center stage. Annu Rev Plant Biol 2008; 59: 573-594.

6 Corbesier L, Vincent C, Jang S, Fornara F, Fan Q, Searle I et al. FT protein movement contributes to long-distance signaling in floral induction of Arabidopsis. Science 2007; 316: 1030-1033.

7 Li C, Gu M, Shi N, Zhang H, Yang X, Osman T et al. Mobile FT mRNA contributes to the systemic florigen signalling in floral induction. Sci Rep 2011; 1: 73.

8 Huang T, Böhlenius H, Eriksson S, Parcy F, Nilsson O. The mRNA of the Arabidopsis gene $F T$ moves from leaf to shoot apex and induces flowering. Science 2005; 309: 1694-1696. 
9 Aki T, Shigyo M, Nakano R, Yoneyama T, Yanagisawa S. Nano scale proteomics revealed the presence of regulatory proteins including three FT-like proteins in phloem and xylem saps from rice. Plant Cell Physiol 2008; 49: 767-790.

10 Bradley D, Ratcliffe O, Vincent C, Carpenter R, Coen E. Inflorescence commitment and architecture in Arabidopsis. Science 1997; 275: 80-83.

11 Mimida N, Goto K, Kobayashi Y, Araki T, Ahn JH, Weigel D et al. Functional divergence of the TFL1-like gene family in Arabidopsis revealed by characterization of a novel homologue. Genes Cells 2001; 6: 327-336.

12 Yamaguchi A, Kobayashi Y, Goto K, Abe M, Araki T. TWIN SISTER OF FT (TSF) acts as a floral pathway integrator redundantly with FT. Plant Cell Physiol 2005; 46: 1175-1189.

13 Yoo SY, Kardailsky I, Lee JS, Weigel D, Ahn JH. Acceleration of flowering by overexpression of MFT (MOTHER OF FT AND TFL1). Mol Cells 2004; 17: 95-101.

14 Komiya R, Yokoi S, Shimamoto K. A gene network for long-day flowering activates RFT1 encoding a mobile flowering signal in rice. Development 2009; 136: 3443-3450.

15 Tamaki S, Matsuo S, Wong HL, Yokoi S, Shimamoto K. Hd3a protein is a mobile flowering signal in rice. Science 2007; 316: 1033-1036.

16 Pin PA, Benlloch R, Bonnet D, Wremerth-Weich E, Kraft T, Gielen JJ et al. An antagonistic pair of $F T$ homologs mediates the control of flowering time in sugar beet. Science 2010; 330: 1397-1400.

17 Lifschitz E, Eviatar T, Rozman A, Shalit A, Goldshmidt A, Amsellem Z et al. The tomato $F T$ ortholog triggers systemic signals that regulate growth and flowering and substitute for diverse environmental stimuli. Proc Natl Acad Sci USA 2006; 103: 6398-6403.

18 Meng X, Muszynski MG, Danilevskaya ON. The FT-like ZCN8 gene functions as a floral activator and is involved in photoperiod sensitivity in maize. Plant Cell 2011; 23: 942-960.

19 Sun H, Jia Z, Cao D, Jiang B, Wu C, Hou W et al. GmFT2a, a soybean homolog of FLOWERING LOCUS $T$, is involved in flowering transition and maintenance. Plos One 2011; 6: e29238.

20 Navarro C, Abelenda JA, Cruz-Oró E, Cuéllar CA, Tamaki S, Silva J et al. Control of flowering and storage organ formation in potato by FLOWERING LOCUS T. Nature 2011; 478: 119-122.

21 Lejeune P, Bernier G, Kinet J-M. Sucrose levels in leaf exudate as a function of floral induction in the long day plant Sinapsis alba. Plant Physiol Bioch 1991; 29: 153-157.

22 Madhusudanan K, Nandakumar S. Carbohydrate changes in shoot tip and subtending leaves during ontogenetic development of pineapple. Zeitschrift für Pflanzenphysiologie 1983; 110: 429-438.

23 Eriksson S, Böhlenius $\mathrm{H}$, Moritz T, Nilsson O. GA4 is the active gibberellin in the regulation of $L E A F Y$ transcription and Arabidopsis floral initiation. Plant Cell 2006; 18: $2172-2181$.

24 Lejeune $\mathrm{P}$, Bernier G, Requier M-C, Kinet J-M. Sucrose increase during floral induction in the phloem sap collected at the apical part of the shoot of the longday plant Sinapis alba L. Planta 1993; 190: 71-74.

25 Houssa P, Bernier G, Kinet J. Qualitative and quantitative analysis of carbohydrates in leaf exudate of the short-day plant, Xanthium strumarium L. during floral transition. J Plant Physiol 1991; 138: 24-28.

26 ElLithy ME, Reymond M, Stich B, Koornneef M, Vreugdenhil D. Relation among plant growth, carbohydrates and flowering time in the Arabidopsis Landsberg erecta $\times$ Kondara recombinant inbred line population. Plant Cell Environ 2010; 33: 1369-1382.

$27 \mathrm{Yu}$ CL, Zhou CM, Zhang TQ, Lian H, Sun Y, Wu J et al. Sugar is an endogenous cue for juvenile-to-adult phase transition in plants. Elife 2013; 2: e00269.

28 Zheng C, Kim K, Matsui S. Effect of brushing on dwarfing, branching and flowering of Dendramthema indicum var. hortense. Hortic Res 2004; 3: 291-295.

29 Ren L, Liu T, Cheng Y, Sun J, Gao J, Dong B et al. Transcriptomic analysis of differentially expressed genes in the floral transition of the summer flowering chrysanthemum. BMC genomics 2016; 17: 673.

30 Oda A, Narumi T, Li T, Kando T, Higuchi Y, Sumitomo K et al. CsFTL3, a chrysanthemum FLOWERING LOCUS T-like gene, is a key regulator of photoperiodic flowering in chrysanthemums. J Exp Bot 2012; 63: 1461-1477.
31 Higuchi Y, Narumi T, Oda A, Nakano Y, Sumitomo K, Fukai S et al. The gated induction system of a systemic floral inhibitor, antiflorigen, determines obligate short-day flowering in chrysanthemums. Proc Natl Acad Sci USA 2013; 110: 17137-17142.

32 Gu C, Zhang X, Jiang J, Guan Z, Zhao S, Fang W et al. Chrysanthemum CmNAR2 interacts with CINNRT2 in the control of nitrate uptake. Sci Rep 2014; 4: 5833-5833.

33 Bustin SA, Benes V, Garson JA, Hellemans J, Huggett J, Kubista M et al. The MIQE guidelines: minimum information for publication of quantitative real-time PCR experiments. Clin Chem 2009; 55: 611-622.

$34 \mathrm{Hsu}$ CY, Adams JP, Kim H, No K, Ma C, Strauss SH et al. FLOWERING LOCUS T duplication coordinates reproductive and vegetative growth in perennial poplar. Proc Natl Acad Sci USA 2011; 108: 10756-10761.

35 Karimi M, Inze D, Depicker A. GATEWAY vectors for Agrobacterium-mediated plant transformation. Trends Plant Sci 2002; 7: 193-195.

36 Yoo SK, Chung KS, Kim J, Lee JH, Hong SM, Yoo SJ et al. CONSTANS activates SUPPRESSOR OF OVEREXPRESSION OF CONSTANS 1 through FLOWERING LOCUS T to promote flowering in Arabidopsis. Plant Physiol 2005; 139: 770-778.

37 Clough SJ, Bent AF. Floral dip: a simplified method for Agrobacterium-mediated transformation of Arabidopsis thaliana. Plant J 1998; 16: 735-743.

38 Shchennikova AV, Shulga OA, Immink R, Skryabin KG, Angenent GC. Identification and characterization of four chrysanthemum MADS-box genes, belonging to the APETALA1/FRUITFULL and SEPALLATA3 subfamilies. Plant Physiol 2004; 134: 1632-1641.

39 Rosso MG, Li Y, Strizhov N, Reiss B, Dekker K, Weisshaar B. An Arabidopsis thaliana T-DNA mutagenized population (GABI-Kat) for flanking sequence tag-based reverse genetics. Plant Mol Biol 2003; 53: 247-259.

40 Hanzawa Y, Money T, Bradley D. A single amino acid converts a repressor to an activator of flowering. Proc Natl Acad Sci USA 2005; 102: 7748-7753.

41 Moghaddam MRB, Ende WVD. Sugars, the clock and transition to flowering. Front Plant Sci 2012; 4: 1-6.

42 Yang L, Xu M, Koo Y, He J, Poethig RS. Sugar promotes vegetative phase change in Arabidopsis thaliana by repressing the expression of MIR156A and MIR156C. Elife 2013; 2: e00260.

43 Ohto M, Onai K, Furukawa Y, Aoki E, Araki T, Nakamura K. Effects of sugar on vegetative development and floral transition in Arabidopsis. Plant Phythol 2001; 127: 252-261.

44 Wahl V, Ponnu J, Schlereth A, Arrivault S, Langenecker T, Franke A et al. Regulation of flowering by trehalose-6-phosphate signaling in Arabidopsis thaliana. Science 2013; 339: 704-707.

45 Harig L, Beinecke FA, Oltmanns J, Muth J, Muller O, Ruping B et al. Proteins from the FLOWERING LOCUS T-like subclade of the PEBP family act antagonistically to regulate floral initiation in tobacco. Plant J 2012; 72: 908-921.

46 Chardon F, Damerval C. Phylogenomic analysis of the PEBP gene family in cereals. J Mol Evol 2005; 61: 579-590.

47 Pin $\mathrm{P}$, Nilsson $\mathrm{O}$. The multifaceted roles of FLOWERING LOCUS T in plant development. Plant Cell Environ 2012; 35: 1742-1755.

48 Ahn JH, Miller D, Winter VJ, Banfield MJ, Lee JH, Yoo SY et al. A divergent external loop confers antagonistic activity on floral regulators FT and TFL1. EMBO J 2006; 25: 605-614.

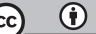

This work is licensed under a Creative Commons Attribution 4.0 International License. The images or other third party material in this article are included in the article's Creative Commons license, unless indicated otherwise in the credit line; if the material is not included under the Creative Commons license, users will need to obtain permission from the license holder to reproduce the material. To view a copy of this license, visit http://creativecommons.org/licenses/ by/4.0/

(c) The Author(s) 2017

Supplementary Information for this article can be found on the Horticulture Research website (http://www.nature.com/hortres) 